\title{
Locally Varying Hubble Parameter in Terms of Reduced Friedmann Equation
}

\author{
Ayman Kassem \\ Egyptair, Cairo, Egypt \\ Email: Physicalmodel2017@gmail.com
}

How to cite this paper: Kassem, A. (2021) Locally Varying Hubble Parameter in Terms of Reduced Friedmann Equation. International Journal of Astronomy and Astrophysics, 11, 175-189.

https://doi.org/10.4236/ijaa.2021.112010

Received: March 29, 2021

Accepted: May 28, 2021

Published: May 31, 2021

Copyright (อ 2021 by author(s) and Scientific Research Publishing Inc. This work is licensed under the Creative Commons Attribution International License (CC BY 4.0).

http://creativecommons.org/licenses/by/4.0/

\begin{abstract}
Assessment of the Hubble parameter as an indicator of the expansion rate of the universe holds a central position in the field of astronomy. From its initial estimate of about $500 \mathrm{~km} \cdot \mathrm{sec}^{-1} \cdot \mathrm{parsc}^{-1}$, this value had been steadily amended as the observational tools became more accurate and precise. Despite this, a gap remains between the value of observations relating to local and nonlocal estimations of the Hubble parameter that gave rise to what became known as the Hubble tension. This tension is addressed here while dealing with space fabric as a cosmological fluid that undergoes transition.
\end{abstract}

\section{Keywords}

Cosmic Fluid Transition, Locally Varying Hubble Parameter, Energy Degrees of Freedom

\section{Introduction}

The $\Lambda$ CDM model which represents standard cosmology had a remarkable success in matching the theoretical predictions of the 6-parameter model to the observational findings.

However, there had been many recent challenges to the integrity of this model in the form of local estimations of the Hubble parameter [1] [2] [3] [4] which have statistically significant difference from those values estimated by non-local measurements, mainly through CMB observations [5] [6].

In addition, the presence of enhanced lensing amplitude whose value exceeds the theoretical prediction $\left(A_{L}>1\right)$ [7] [8] is interpreted as an indication of a closed universe-in contradiction to the basic assumption of the $\Lambda \mathrm{CDM}$ model of a flat cosmology.

Here we will try to address those two questions concerning the Hubble ten- 
sion and the curvature tension which were tackled previously [9] using degree of freedom approach, but this time the Friedmann equations would be employed.

\section{Hubble Parameter-Radius of the Universe $\left(r_{u e}\right)$ Relationship}

The Hubble parameter is related to the universe radius via the scalar factor as follows

$$
r_{\text {ue }}=a(t) r_{\text {ueo }}
$$

where $r_{\text {ue }}, r_{\text {ueo }}$ denote the radius of the universe at any time $(t)$ and at present time $\left(t_{o}\right)$

$$
\begin{array}{r}
\frac{\mathrm{d} r_{\text {ue }}}{\mathrm{d} t}=r_{\text {uеo }} \frac{\mathrm{d} a(t)}{\mathrm{d} t} \\
H=\frac{\frac{\mathrm{d} a(t)}{\mathrm{d} t}}{a(t)}=\frac{r_{\text {uеo }} \frac{\mathrm{d} a(t)}{\mathrm{d} t}}{r_{\text {uеo }} a(t)}=\frac{\frac{\mathrm{d} r_{\text {ue }}(t)}{\mathrm{d} t}}{r_{\text {uе }}(t)}
\end{array}
$$

\section{Decelerating Pressure-Less Universe}

This analysis is based on a model for quanton fields [10], which are space and time varying, when interacting they generate inflationary and gravitational interactions of space fabric which are at origin of dark energy and dark matter.

Here, the standard form of Friedmann equations will be tackled, while later on a slightly modified version will be introduced. Friedman acceleration equation is written as

$$
\begin{gathered}
\frac{r_{u e}^{\prime \prime}}{r_{u e}}=-\frac{4 \pi G}{3}\left(\varrho_{m}+\frac{3 p}{c^{2}}\right) \\
\varrho_{e} \text { : energy density }=\frac{K_{\varrho}}{t^{2}} \\
r_{u e}=K_{r u} t^{\frac{2}{3}}
\end{gathered}
$$

where mass density is defined as

$$
\varrho_{m}=\frac{\varrho_{e}^{2}}{c^{2}}
$$

$K_{\varrho}, K_{r u}$ : constants of proportionality [9]. For the case of pressure-less fluid this reduces to

$$
\frac{r_{u e}^{\prime \prime}}{r_{u e}}=-\frac{4 \pi G \varrho_{m}}{3}
$$

Given that

$$
\begin{gathered}
r_{u e}=K_{r u} t^{\frac{2}{3}} \\
r_{u e}^{\prime}=\frac{\mathrm{d} r_{u e}}{\mathrm{~d} t}=\frac{2}{3} K_{r u} t^{\frac{-1}{3}}
\end{gathered}
$$

And 


$$
\varrho_{m}=\frac{k_{\varrho}}{c^{2} t^{2}}
$$

Substituting with this equivalent value for $r_{u e}$

$$
\begin{gathered}
r_{u e}^{\prime \prime}=-\frac{4 \pi G \varrho_{m}}{3} r_{u e}=-\frac{4 \pi G k_{\varrho}}{3 c^{2} t^{2}} r_{u e} \\
t=\left(\frac{r_{u e}}{k_{r u}}\right)^{\frac{3}{2}}, \frac{1}{t^{2}}=\left(\frac{k_{r u}}{r_{u e}}\right)^{3}
\end{gathered}
$$

substituting in (8-3) with value of $\frac{1}{t^{2}}$ and integrating w.r.t $r_{\text {ue }}$

$$
\begin{gathered}
\int r_{u e}^{\prime \prime} \mathrm{d} r_{u e}=-\int \frac{4 \pi G k_{\varrho}}{3 c^{2}}\left(\frac{k_{r u}}{r_{u e}}\right)^{3} r_{u e} \mathrm{~d} r_{u e} \\
\int r_{u e}^{\prime \prime} \mathrm{d} r_{u e}=-\int \frac{4 \pi G k_{\varrho} k_{r u}^{3}}{3 c^{2} r_{u e}^{2}} \mathrm{~d} r_{u e} \\
\int \frac{\mathrm{d}\left(r_{u e}^{\prime}\right)}{\mathrm{d} t} \mathrm{~d} r_{u e}=\int \frac{\mathrm{d} r_{u e}^{\prime}}{\mathrm{d} r_{u e}} \frac{\mathrm{d} r_{u e}}{\mathrm{~d} t} \mathrm{~d} r_{u e}=\int \frac{\mathrm{d} r_{u e}}{\mathrm{~d} t} \mathrm{~d} r_{u e}^{\prime}=\int r_{u e}^{\prime} \mathrm{d} r_{u e}^{\prime}=\frac{\left(r_{u e}^{\prime}\right)^{2}}{2} \\
\left(r_{u e}^{\prime}\right)^{2}=\frac{8 \pi G k_{\varrho} k_{r u}^{3}}{3 c^{2} r_{u e}}+\text { constant } \\
\frac{\left(r_{u e}^{\prime}\right)^{2}}{\left(r_{u e}\right)^{2}}=\frac{8 \pi G k_{\varrho} k_{r u}^{3}}{3 c^{2} r_{u e}^{3}}+\text { constant }
\end{gathered}
$$

Given that $\frac{k_{r u}^{3}}{r_{u e}^{3}}=\frac{1}{t^{2}}$

$$
\frac{\left(r_{u e}^{\prime}\right)^{2}}{\left(r_{u e}\right)^{2}}=H^{2}=\frac{8 \pi G \varrho_{m}}{3}+\text { constant }
$$

The constant of integration equals $\frac{-k c^{2}}{r_{u e}^{2}}$, which is the usual form of Friedmann equation. If the curvature term is small enough to be neglected in comparison to density term.

$$
\left(\frac{r_{u e}^{\prime}}{r_{u e}}\right)^{2}=\frac{8 \pi G \varrho_{m}}{3 c^{2}}
$$

which is the first Friedmann equation of Einstein DeSitter model for flat matter dominated universe this time in a closed cosmology model.

Now the Hubble parameter

$$
H=\frac{\frac{\mathrm{d} r_{u e}(t)}{\mathrm{d} t}}{r_{u e}(t)}=\frac{\frac{2}{3} K_{r u} t^{\frac{-1}{3}}}{K_{r u} t^{\frac{2}{3}}}=\frac{2}{3 t}
$$

Back to the first Friedmann equation and from before

$$
\frac{8 \pi G \varrho_{m}}{3}=\frac{4}{9 t^{2}}=H^{2}
$$

The closed inflationary model of the nature $r_{u e}=K_{r u}=t^{\frac{2}{3}}$ of a four dimensional energy density translates into 3 dimensional mass density of the type 
$\varrho_{m}=\frac{K}{a^{3}}$. As for the three dimensional universe, the four degrees of freedom of expanding energy density are the result of adding three degrees of freedom for the qunanton volume in addition to one degree of freedom due to quanton splitting [9], and when considering length, its $\frac{4}{3}$ degrees of freedom are the result of adding one degree of freedom of the quanton radius and $\left(\frac{1}{3}\right)$ degrees of freedom as a result of quanton splitting.

In this closed inflationary model, the energy density plays the pivotal role due to its relationship with the energy degree of freedom as this was the major parameter which controlled the inflationary process and contributed to the uniformity and the homogeneity of space fabric as it is today.

Despite being a closed model, it had a clear matter density term dominance over curvature term especially in the earliest part of inflationary history.

The relative ratio between the density term $\left(\frac{8 \pi G \varrho_{m}}{3}\right)$ and the curvature term $\left(\frac{c^{2}}{r_{u e}^{2}}\right)$ is as follows

$$
R R=\frac{\frac{8 \pi G \varrho_{m}}{3}}{\frac{c^{2}}{r_{u e}^{2}}}=\frac{8 \pi G K_{\varrho}}{3 c^{2} t^{2}} * \frac{K_{r u}^{2} t^{\frac{4}{3}}}{c^{2}}=\frac{8 \pi G K_{\varrho} K_{r u}^{2}}{3 c^{4} t^{\frac{2}{3}}}
$$

The inflationary process of the universe started with a dominant density term in comparison to the curvature term with a relative ratio $R R$ of almost $1: 2 \times 10^{22}$ (at $t=t_{\text {Planck }}$ ).

Gradually the curvature term assumed greater relative importance, and this gradual loss of dominance for the density term can be understood as energy density expansion is expressed as $\varrho_{e}=\frac{k_{\varrho}}{t^{2}}$ in other words energy density inversely proportional to true time squared $\left(t^{2}\right)$, while the radius of curvature is put as $r_{u e}=k_{r u} t^{\frac{2}{3}}$ as the curvature term $\left(\frac{c^{2}}{r_{u e}^{2}}\right)$ is inversely proportional to $\left(t^{\frac{4}{3}}\right)$.

The dominance of density term is manifested as a decelerating inflationary regime which weakens as time progresses, and gradually both terms converge. In fact for very small values of $(t)$ the curvature term can be disregarded and this closed inflationary model can be viewed as very similar to flat model.

\section{Transition Time and Afterwards}

The inflation of the universe was accompanied by a reduction of the energy density which led to a weakness of decelerating effect with regard to the rate of change of its radius $\left(\frac{\mathrm{d} r_{u e}}{\mathrm{~d} t}\right)$. The deceleration came to a halt as the rate $\frac{\mathrm{d} r_{u e}}{\mathrm{~d} t}$ approached the value of (c). This happened as the space fabric whose constituent quantons must expand in a degree of freedom manner or in other words $\frac{\mathrm{d} r_{o}}{\mathrm{~d} t}=c$, where $r_{o}$ denotes the outer radius of the universe.

From this point on, the universe radius is expressed in terms of the equivalent sphere whose radius equals $r_{u e}$ (which is also equal to Hubble radius), outer 
and inner radii $r_{o}, r_{i}$ of a shell.

The transition from sphere to shell shaped universe is the result of a universe volume $V_{u}$ being smaller than volume encompassed by the outer shell $V_{o}$, since the rate of generation of this volume $\frac{\mathrm{d} V_{u}}{\mathrm{~d} t}$ is smaller than the rate of increase of the outer sphere volume $\frac{\mathrm{d} V_{o}}{\mathrm{~d} t} \quad[9]$.

The quantons now forth behave as a quantum relativistic (photon like) gas (keeping in mind that photons were defined as relativistic quantons) [10] which obey the relationship

$$
3 p=\frac{U}{V}=\varrho_{e}[11][12]
$$

The Friedmann acceleration equation

$$
\frac{r_{u e}^{\prime \prime}}{r_{u e}}=-\frac{4 \pi}{3}\left(\varrho_{m}+\frac{3 p}{c^{2}}\right)
$$

During post transition era, the pressure has an accelerating effect which nullifies the decelerating gravitational effect of density term.

In other words

$$
\frac{3 p}{c^{2}}=-\varrho_{m}
$$

and consequently

$$
\frac{r_{u e}^{\prime \prime}}{r_{u e}}=\text { zero }, \text { or } r_{u e}^{\prime \prime}=\text { zero }
$$

which leads to $r_{u e}^{\prime}=$ constant $=c$.

Under such conditions the second Hubble equation becomes

$$
H^{2}=\frac{c^{2}}{r_{u e}^{2}}
$$

$r_{u e}$ : the equivalent radius of the universe.

At present an equivalent universe radius equals $1.36 \times 10^{26}$ meters and can be expressed as

$$
r_{u e}=c t_{H}
$$

$t_{H}=$ Hubble time and this relationship is valid for $t>t_{r}$. Figure 1 represents the

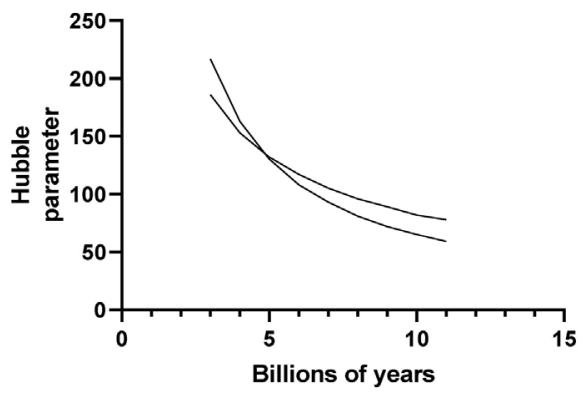

Figure 1. Representation of Hubble parameter transition from pressure-less fluid of the nature $H_{0}=\frac{2}{3 t}$ to the relativistic fluid of the nature $H_{0}=\frac{c}{r_{u e}}$. 
transition from a decelerating fluid (the higher curve on the left) to a relativistic fluid (the lower curve on the left) where the transition time is the instance when the Hubble parameters of the two flow regimes were momentarily equal.

\section{Local Hubble Parameter}

Possible explanations for the inconsistency between the local and the universal values of the Hubble parameter, apart from systematic errors, range from early dark energy [13] [14], acoustic dark energy [15], modified gravitation [16], Dynamic dark energy [17] [18], and neutrino physics [19].

This model proposed an inflationary scenario that transits from sphere to shell shaped universe [9], under such a model the Hubble parameter can have a local value, while its universal value can still be assessed via non local phenomena like CMB.

Previously, the values of the Hubble parameter were obtained in terms of the outer $\left(r_{o}\right)$, inner radius $\left(r_{i}\right)$, and their rates of change with time, the same methodology can be elaborated further to allow for the determination of the Hubble parameter at any radius $\left(r_{x}\right)$.

Remembering that

$$
\begin{gathered}
r_{o}(t)=c\left(t-t_{r}\right)+K_{r u} t_{r}^{\frac{2}{3}} \\
\frac{\mathrm{d} r_{o}}{\mathrm{~d} t}=c
\end{gathered}
$$

$t_{r}:$ transition time

$$
\begin{gathered}
r_{i}(t)=\sqrt[3]{r_{o}^{3}-\frac{3}{4 \pi} V_{u}} \\
\frac{\mathrm{d} r_{i}}{\mathrm{~d} r_{o}}=\frac{1}{r_{i}^{2}}\left(r_{o}^{2}-\frac{1}{4 \pi c} \frac{\mathrm{d} V_{u}}{\mathrm{~d} t}\right) \\
\frac{\mathrm{d} r_{i}}{\mathrm{~d} t}=\frac{\mathrm{d} r_{o}}{\mathrm{~d} t} \frac{\mathrm{d} r_{i}}{\mathrm{~d} r_{o}}=c \frac{\mathrm{d} r_{i}}{\mathrm{~d} r_{o}}
\end{gathered}
$$

Now for the Hubble parameter an any point on the radius $r_{x}$.

Given that $r_{o}>r_{x}>r_{i}$ and

$$
\begin{gathered}
x=\frac{r_{x}-r_{i}}{r_{o}-r_{i}} \\
r_{x}=x\left(r_{o}-r_{i}\right)+r_{i}=r_{o}-(1-x)\left(r_{o}-r_{i}\right)
\end{gathered}
$$

After rearrangement

$$
r_{x}=x r_{o}+(1-x) r_{i}
$$

When differentiating w.r.t time

$$
\frac{\mathrm{d} r_{x}}{\mathrm{~d} t}=x \frac{\mathrm{d} r_{o}}{\mathrm{~d} t}+(1-x) \frac{\mathrm{d} r_{i}}{\mathrm{~d} t}
$$


And the local Hubble parameter

$$
\begin{gathered}
H(x)=\frac{\frac{\mathrm{d} r_{x}}{\mathrm{~d} t}}{r_{x}}=\frac{x \frac{\mathrm{d} r_{o}}{\mathrm{~d} t}+(1-x) \frac{\mathrm{d} r_{i}}{\mathrm{~d} t}}{r_{x}} \\
H(x)=\frac{x \frac{\mathrm{d} r_{o}}{\mathrm{~d} t}+(1-x) \frac{\mathrm{d} r_{i}}{\mathrm{~d} t}}{x\left(r_{o}-r_{i}\right)+r_{i}}=\frac{x \frac{\mathrm{d} r_{o}}{\mathrm{~d} t}+(1-x) \frac{\mathrm{d} r_{i}}{\mathrm{~d} t}}{x r_{o}+(1-x) r_{i}}
\end{gathered}
$$

Figure 2 charts the variation of the Hubble parameter along the radial distance $r_{x}$.

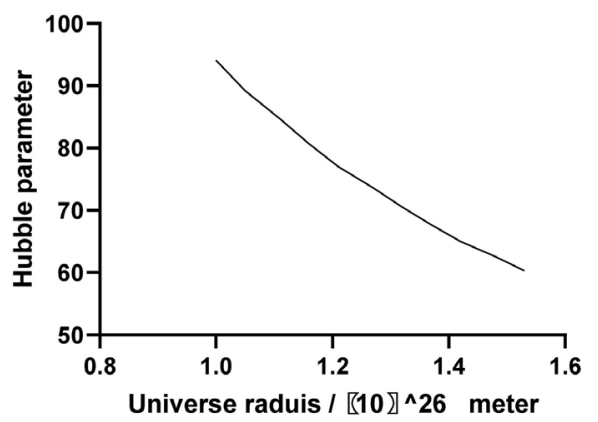

Figure 2. Representation for the variation of the local value of the Hubble parameter along the radial direction $r_{x}, r_{o}>r_{x}>r_{i}$.

\section{Newtonian Interpretation of the Transition Time}

Based on the Newtonian concept of gravitation [20] [21] an illustration can be made about the physical meaning of each of Friedmann equations, given that the mass of the universe $M_{u}$, the second Newton law is written as

$$
\frac{\mathrm{d}^{2} r_{u e}}{\mathrm{~d} t^{2}}=-\frac{G M_{u}}{r_{u e}^{2}}
$$

After multiplying both terms by $\frac{\mathrm{d} r_{u e}}{\mathrm{~d} t}$ and integrating, the energy equation is obtained.

$$
\frac{1}{2}\left(\frac{\mathrm{d} r_{u e}}{\mathrm{~d} t}\right)^{2}=\frac{G M_{u}}{r_{u e}}+U
$$

where the density term represents the gravitational potential energy and the constant $(U)$ represents the total energy of the universe, while the time derivative term represents the kinetic energy.

For the case of the symmetrically expanding universe the mass can be expressed as $M_{u}=\frac{4 \pi \varrho_{e} r_{u e}^{3}}{3}$ when dividing by $r_{u e}^{2}$.

In pre-transition era the kinetic energy equaled the gravitational decelerating potential, while in post transition universe total energy is represented by the kinetic energy as there was no decelerating potential.

The kinetic energy of the universe is transformed from being equal to the gravitational potential to being equal to the total energy in the universe in the post 
transition era.

\section{The Dual Nature of Density Term}

The motivation behind the following analysis is to study how the cosmological Transition took place given that it must have been a smooth one between the two epochs. The condition of such smoothness is that both the density and the curvature terms must momentarily be equal at the instant of transition. Previously, it was shown that the density term had a relative dominance in magnitude over the curvature term. The curvature term approaches the density term but not at any point the universe's inflationary history did both terms become equal. Under such conditions the cosmological transition took place under a modified gravitation. Here a distinction is made between the energy density spatial expansion (whose pattern is uniform throughout the inflationary history of the universe), and the volumetric expansion which is altered at the transition instance. Previously the universe radius was defined as

$$
r_{u e}=k_{r u} t^{\frac{2}{3}}
$$

where the degrees of freedom corresponding to $\left(r_{u e}\right)$ are

$$
\operatorname{Dof}_{r_{u e}}=\frac{4}{3}
$$

This allows universe radius to be put in the form

$$
r_{u e}=k_{r u} t^{\frac{D o f_{u e}}{2}}
$$

Similarly for the case of spatial expansion which corresponds to the two dimensional expansion of the quanton fields and the spatial radius is represented by $r_{s}$, the degrees of freedom corresponding to the spatial radius

$$
\begin{gathered}
\operatorname{Dof}_{r_{s}}=\frac{4}{2}=2 \\
r_{s}=k_{r s} t^{\frac{D o f_{r_{s}}}{2}}=k_{r s} t
\end{gathered}
$$

The value of $k_{r s}$ equals the constant (c) as the spatial expansion corresponds a degree of freedom expansion of the quanton energy fields.

The spatial expansion is based on Hubble parameter of the discrete quanton Fields.

$$
\begin{gathered}
r_{s}=c t \\
v=\frac{\mathrm{d} r_{s}}{\mathrm{~d} t}=c
\end{gathered}
$$

The spatial expansion based Hubble parameter $\left(H_{s}\right)$ can then be found as follows

$$
H_{s}=\frac{\frac{\mathrm{d} r_{s}}{\mathrm{~d} t}}{r_{s}}=\frac{c}{c t}=\frac{1}{t} \quad(t \text { true time })
$$

Now the modified Friedmann equation becomes 


$$
H_{s}^{2}=\frac{8 \pi G \varrho_{m}}{3}=\frac{1}{t^{2}}
$$

This equation remains time invariant. (pre- and post-transition), and this is understandable as the expansion nature of quanton fields remains constant irrespective of the inflationary nature of the universe.

The Friedman equation for the volumetric expansion in a pre-transition world now becomes

$$
H_{v}^{2}=\left(\frac{2}{3 t}\right)^{2}=\left(\frac{2}{3}\right)^{2} \frac{8 \pi G \varrho_{m}}{3}
$$

$H_{v}:$ Hubble parameter for the volumetric expansion and the ratio between the volumetric and spatial expansion Hubble parameters for the pre-transition era

$$
\frac{H_{v}}{H_{s}}=\frac{t}{t_{H}}=\frac{2}{3}
$$

This ratio between the volumetric and the spatial expansion Hubble parameters (as well as the ratio between Hubble time and true time) was a constant one during the pre-transition era as it was a case of inflation into a spherically shaped universe.

In pre-transition time the density term played both roles, namely volumetric and spatial expansion, however this duality came to an end with the advent of the transition and afterwards, as the curvature term assumed the role of volumetric expansion while the density term maintained the role of spatial expansion and the ratio between the two in post transition era is no longer a constant one as it was

$$
\begin{aligned}
\frac{H_{v}}{H_{s}}= & \frac{\frac{c}{\sqrt[3]{\frac{3}{4 \pi} k_{v t^{2}}}}}{\frac{1}{t}}=\frac{t}{t_{H}} \\
= & \frac{c t^{\frac{1}{3}}}{\sqrt[3]{\frac{3}{4 \pi} k_{v u}}}
\end{aligned}
$$

where the universe volume is defined as

$$
V_{u}=k_{v u} t^{2}[9]
$$

and its equivalent radius

$$
r_{u e}=\left[\sqrt[3]{\frac{3}{4 \pi} k_{v u}}\right] t^{\frac{2}{3}}
$$

and the volumetric Hubble parameter of the post-transition world now becomes

$$
H_{v}=\frac{c}{r_{u e}}
$$

Previously the value of the vacuum energy density and the total energy content of the universe was provided based on empirical data [22] [23]. Now more 
refined value for both can be determined based on (9-7), which yields the following values of vacuum energy density and total energy content of the universe respectively.

$$
\begin{gathered}
\varrho_{e}=\frac{3 c^{2}}{8 \pi G}=8.45 \times 10^{-10} \mathrm{j} \cdot \mathrm{m}^{-3} \\
E_{u}=8.9 \times 10^{69} \text { joules }
\end{gathered}
$$

Equation (9-7) allows for the following

$$
\begin{aligned}
3 c^{2} & =8 \pi G k_{\varrho} \\
k_{\varrho} & =\frac{3 c^{2}}{8 \pi G}
\end{aligned}
$$

For a closed universe spatially symmetric universe,

$$
\begin{gathered}
V_{u}=\frac{E_{u}}{\varrho_{e}} \\
r_{u e}=\sqrt[3]{\frac{3 E_{u}}{4 \pi \varrho_{e}}}=\sqrt[3]{\frac{3 E_{u}}{4 \pi k_{\varrho}} t^{\frac{2}{3}}=k_{r u} t^{\frac{2}{3}}}
\end{gathered}
$$

Substituting with value of $k_{\varrho}$ from (20-7)

$$
\begin{gathered}
k_{r u}=\sqrt[3]{\frac{3 * 8 \pi G E_{u}}{4 \pi * 3 c^{2}}}=\sqrt[3]{\frac{2 G E_{u}}{c^{2}}} \\
r_{u e}=\sqrt[3]{\frac{2 G E_{u}}{c^{2}}} t^{\frac{2}{3}} \\
V_{u}=\frac{8 \pi G E_{u}}{3 c^{2}} t^{2}
\end{gathered}
$$

Equations $(23,24,25-7)$ are very important as they relate the cosmological parameters in terms of the fundamental physical constants $G, c$ in the absence of the related time constants (k's).

The concept of uniform density expansion of the form $\frac{8 \pi G \varrho_{e}}{3 c^{2}}=\frac{1}{t^{2}}$ allows for the development of more refined values of actual energy density while maintaining the symmetries of the quanton physical and cosmological ratios as illustrated in the supportive data.

Figure 3 displays relationship between true time and Hubble time which is based on Equations (10-7) and (16-7) for pre and post transition respectively.

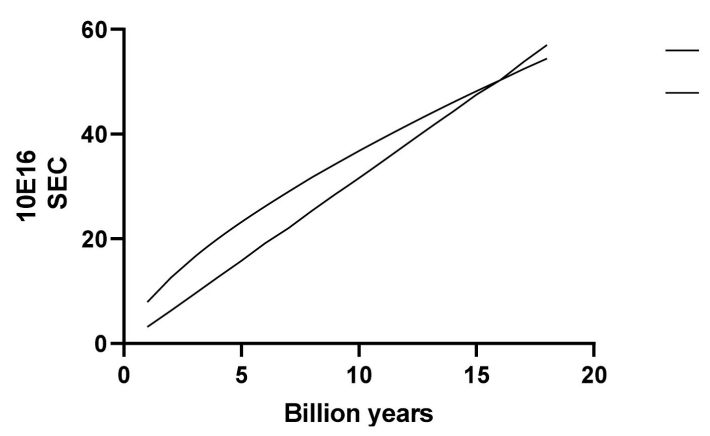

Figure 3. The extrapolated relationship between true time and Hubble time. 
Note that the intersection point where the two values are equal marks another possible transition when density term and curvature term are equal in value.

Table 1 offers the modified version of the Friedmann equation for the spatial and volumetric expansion.

Table 1. The density term represents both the volumetric and the spatial expansion in the pre-transition Era while it represents only the spatial expansion in post transition era.

\begin{tabular}{lc}
\hline \multicolumn{1}{c}{ Era } & \multicolumn{1}{c}{ expression } \\
\hline $\begin{array}{l}\text { Pre-transition } \\
\text { Spatial expansion }\end{array}$ & $H_{s}^{2}=\frac{8 \pi G \varrho_{m}}{3}=\frac{1}{t^{2}}$ \\
$\begin{array}{l}\text { Pre-transition } \\
\text { Volumetric expansion }\end{array}$ & $H_{v}^{2}=\left(\frac{2}{3 t}\right)^{2}=\left(\frac{2}{3}\right)^{2}\left(\frac{8 \pi G \varrho_{m}}{3}\right)$ \\
$\begin{array}{l}\text { Post transition } \\
\text { Spatial expansion }\end{array}$ & $H_{s}^{2}=\frac{8 \pi G \varrho_{m}}{3}=\frac{1}{t^{2}}$ \\
$\begin{array}{l}\text { Post transition } \\
\text { volumetric expansion }\end{array}$ & $H_{v}=\frac{c}{r_{u e}} \quad\left(r_{\text {ue }}\right.$ : equivalent spherical radius $)$ \\
\hline
\end{tabular}

The value of Hubble time is greater than true time up till now, and consequently, the value of the term $\left(\frac{1}{t^{2}}\right)$ is bound to be greater than the value of the term $\left(\frac{1}{t_{H}^{2}}\right)$, therefore, the actual mass density is always greater than the critical density.

Based on the previous conclusion the value of the curvature parameter can be obtained

$$
\begin{gathered}
\Omega=\frac{\varrho_{m}}{\varrho_{m c}}=\frac{t_{H}^{2}}{t^{2}}=\frac{8.45 \times 10^{-10}}{7.74 \times 10^{-10}}=1.09 \\
\Omega_{k}=1-\Omega=-0.09
\end{gathered}
$$

An explanation of the near zero value of the curvature parameter as a result of the constraining of observational cosmological parameters [24] lies in the fact that this near zero curvature parameter (which is perceived as an evidence of a flat universe) belongs to a closed universe which is expanding at a rate that equals that of an open universe due to geometric degeneracy which allows various closed models to generate the same observational results as flat model, namely

$$
\begin{gathered}
\varrho_{m c}(\text { perceived critical mass density })=\frac{3 H_{v}^{2}}{8 \pi G} \\
H_{v}^{2}=\left(\frac{c}{r_{u e}}\right)^{2} \quad \text { (actual volumetric expansion) } \\
\frac{8 \pi G \varrho_{m c}}{3}=\left(\frac{c}{r_{u e}}\right)^{2}
\end{gathered}
$$




$$
\varrho_{m c}=\frac{8 \pi G c^{2}}{3 r_{\text {ueo }}^{2}}
$$

\section{Effect of Presence of Normal Matter}

\subsection{Departure from Parameter Ratio Symmetry with Time}

Previous work [9] the presented cosmological inflationary model which the quanton and the cosmological parameters were determined based on their energy degree of freedom. The relative ratios of those parameters between the present day and the Planck Era $\left(R R_{x}\right)$ were deduced in the form

$$
R R_{x}=\left(\frac{t_{0}}{t_{P}}\right)^{\frac{D o f_{x}}{2}}=\left(\frac{4.37 \times 10^{17}}{5.39 \times 10^{-44}}\right)^{\frac{D o f_{x}}{2}}=(2.84)^{\frac{D o f_{x}}{2}}
$$

where $R R_{x}$ is the relative ratio between the value of the parameter $(x)$ at the present day to its value at Planck time given its degrees of freedom (Dof). That model was based on either the absence of the normal matter or considering it to be as diffusive as dark matter. In either case the result was ideal ratios that do not deviate from that of time relative ratio. But in reality the agglomerated, non-diffusive nature of the normal matter reduces its gravitational effect so allowing energy density further expansion and as a result the quanton and the cosmological parameter relative ratios are higher by two or 3 hundredths from that ideal relative ratio.

\subsection{Underestimation of the Energy Density}

The extent of lensing of observational CMB radiation is affected by the matter distribution, as a result the observed curvature parameter $\left(\Omega_{k}=-0.045\right)$ is an underestimation of the actual space curvature while the ideal relationship $\frac{1}{t^{2}}=\frac{8 \pi G \varrho_{m}}{3}$ provides an overestimation of the energy density. As a result of the combination of those effects, the actual curvature parameter is somewhere between the observed value and the ideal value.

\section{Ethical Statement}

The author declares that this work fully complies with the ethical guidelines as had been stated by the journal.

\section{Data Availability}

The data that support the findings of this study are available from the corresponding author, upon request or alternatively it can be downloaded via the following links:

1)

https://docs.google.com/spreadsheets/d/1fc0iVjZHgRcrN2RsezX6ySpjnxcpdRa3 ledit\#gid $=626736170$

2) https://archive.org/details/calculations-100 
3) https://drive.flipdrive.com/drive/NYdIDzfDx1Or737LkQvT9JITRXoYQakM

\section{Discussion}

Though this model adheres to the main outlines of the GR based standard cosmology but amendments were introduced which can be summarized as follows

1) Distinction is being made between spatial and volumetric expansion of energy density due to the nature of quantized two dimensional quanton space and time varying fields expanding in three dimensional space.

2) In the pre-transition era

a) The Hubble parameter is represented by the modified density term only despite being a closed model $(k \neq$ zero as density term is greater in magnitude than curvature term).

b) As a result of this distinction between spatial expansion volumetric expansion, a factor of $\left(\frac{2}{3}\right)$ was introduced to density term to account for this difference when calculating the universal volumetric Hubble parameter.

3) In post transition era, introduction of a locally varying Hubble parameter which seems to be a departure from general principle of cosmology which necessitates that inflation is the same everywhere in the universe, but otherwise the reminder of that principle still holds since the degree of freedom expansion of the statistically distributed quanton densities as well as parameter synchronization ensured the space fabric uniformity and homogeneity.

Finally Equation (9-7) provided a constraint that broke the geometric degeneracy and provided a unique closed inflationary model that corresponds to observational values.

\section{Conclusions}

1) Cosmic transition from decelerating pressure-less fluid to a relativistic fluid allows Hubble parameter to be varied locally, while the universal Hubble parameter can be assessed in terms of curvature term of Friedman equation.

2) Both density and curvature terms carry their own time imprints (true time and Hubble time) which express spatial and volumetric expansion of energy density respectively.

3) Smooth cosmic transition requires that the density and the curvature terms be equal momentarily, this is possible only under modified gravitation.

\section{Acknowledgements}

A preprint of this paper can be found at https://vixra.org/abs/2102.0178.

\section{Conflicts of Interest}

The author declares no conflicts of interest regarding the publication of this paper. 


\section{References}

[1] Riess, A.G., Casertano, S., Yuan, W., Macri, L.M. and Scolnic, D. (2019) Large Magellanic Cloud Cepheid Standards Provide a 1\% Foundation for the Determination of the Hubble Constant and Stronger Evidence for Physics beyond $\Lambda$ CDM. Astrophysical Journal, 876, 85. https://doi.org/10.3847/1538-4357/ab1422

[2] Wong, K.C., Suyu, S.H., Chen, G.F., Rusu, C.L., et al. (2019) H0LiCOW-XIII. A 2.4 per cent Measurement of $\mathrm{H}_{0}$ from Lensed Quasars: 5.3 $\sigma$ Tension between Earlyand Late-Universe Probe. Monthly Notices of the Royal Astronomical Society, 498, 1420-1439. https://doi.org/10.1093/mnras/stz3094

[3] De Jaeger, T., Stahl, B.E., Zheng, W., Filippenko, A.V., Riess, A.G. and Galbany, L. (2020) A Measurement of the Hubble Constant from Type II Supernovae. Monthly Notices of the Royal Astronomical Society, 496, 3402-3411. https://doi.org/10.1093/mnras/staa1801

[4] Huang, C.D., Riess, A.G., Yuan, W., Macri, L.M., Zakamska, N.L., Casertano, S., et al. (2020) Hubble Space Telescope Observations of Mira Variables in the SN Ia Host NGC 1559: An Alternative Candle to Measure the Hubble Constant. The Astrophysical Journal, 889, 5. https://doi.org/10.3847/1538-4357/ab5dbd

[5] Aghanim, N., Akrami, Y., Ashdown, M., et al. (2020) Planck 2018 Results VI. Cosmological Parameters. Astronomy \& Astrophysics, 641, A6.

[6] Aiola, S., Calabrese, E., Maurin, L., Naess, S., Schmitt, B.L., et al. (2020) The Atacama Cosmology Telescope: DR4 Maps and Cosmological Parameters. Journal of Cosmology and Astroparticle Physics, 12, 047. https://doi.org/10.1088/1475-7516/2020/12/047

[7] Aghanim, N., Akrami, Y., Ashdown, M., et al. (2020) Planck 2018 Results. V. CMB Power Spectra and Likelihoods. Astronomy \& Astrophysical Journal, 641, A5.

[8] Ade, P.A.R., Aghanim, N., Arnaud, M., et al. (2016) Planck 2015 Results XIII. Cosmological Parameters. Astronomy \&Astrophysics, 594, A13.

[9] Kassem, A. (2020) Symmetries in the Universe, a Quanton Origin. International Astronomy and Astrophysics Research Journal, 2, 52-70. http://www.journaliaarj.com/index.php/IAARJ/article/view/27

[10] Kassem, A. (n.d.) Quanton Based Model of Field Interactions. http://vixra.org/abs/1912.0314

[11] Das, S. and Roychowdhury, D. (2020) Thermodynamics of a Photon Gas with an Invariant Energy Scale. Physical Review D, 81, Article ID: 085039. http://doi.org/10.1103/PhysRevD.81.085039

[12] Mansuripur, M. and Han, P. (2017) Thermodynamics of Radiation Pressure and Photon Momentum. Dholakia, K. and Spalding, G.C., Eds., Optical Trapping and Optical Micromanipulation XIV, Proceedings of SPIE Vol. 10347, 1-20. http://doi.org/10.1117/12.2274589

[13] Poulin, V., Smith, T.L., Karwal, T. and Kamionkowski, M. (2019) Early Dark Energy Can Resolve the Hubble Tension. Physical Review Letters, 122, 221301. https://doi.org/10.1103/PhysRevLett.122.221301

[14] Niedermann, F. and Sloth, M.S. (2020) Resolving the Hubble Tension with New Early Dark Energy. Physical Review D, 102, Article ID: 063527. https://doi.org/10.1103/PhysRevD.102.063527

[15] Lin, M.X., Benevento, G., Hu, W. and Raveri, M. (2019) Acoustic Dark Energy: Potential Conversion of the Hubble. Physical Review D, 100, 063542.

https://doi.org/10.1103/PhysRevD.100.063542 
[16] Abadi, T. and Kovetz, E.D. (2021) Can Conformally Coupled Modified Gravity Solve the Hubble Tension? Physical Review D, 103, 023530.

https://doi.org/10.1103/PhysRevD.103.023530

[17] Di Valentino, E., Ferreira, R.Z., Visinelli, L. and Danielsson, U. (2019) Late Time Transitions in the Quintessence Field and the H0 Tension. Physics of the Dark Universe, 26, 100385. https://doi.org/10.1016/j.dark.2019.100385

[18] Pan, S., Yang, W., DiValentino, E., Saridakis, E.N. and Chakraborty, S. (2019) Interacting Scenarios with Dynamical Dark Energy: Observational Constraints and Alleviation of the H0 Tension. Physical Review D, 100, 103520.

https://doi.org/10.1103/PhysRevD.100.103520

[19] Lancaster, L., Cyr-Racine, F.-Y., Knox, L. and Pan, Z. (2017) A Tale of Two Modes: Neutrino Free-Streaming in the Early Universe. Journal of Cosmology and Astroparticle Physics, 7, 033. https://doi.org/10.1088/1475-7516/2017/07/033

[20] Tipler, F.J.Rigorous Newtonian cosmologyNewtonian cosmology revisited

[21] Telkamp, H. (2016) Machian Derivation of the Friedmann Equation. Physical Review D, 94, 043520. https://doi.org/10.1103/PhysRevD.94.043520

[22] Oldershaw, R.L. (1989) Towards a Resolution of the Vacuum Energy Density Crisis. https://arxiv.org/abs/0901.3381

[23] Valev, D. (2014) Estimations of Total Mass and Energy of the Universe. Physics International, 5, 15-20. https://doi.org/10.3844/pisp.2014.15.20

[24] Efstathiou, G. and Gratton, S. (2020) The Evidence for a Spatially Flat Universe. Monthly Notices of the Royal Astronomical Society: Letters, 496, L91-L95. https://doi.org/10.1093/mnrasl/slaa093 